Research Article

\title{
A Nonstationary Ternary 4-Point Shape-Preserving Subdivision Scheme
}

\author{
Jieqing Tan ${ }^{1,2}$ and Guangyue Tong $\mathbb{D}^{2}$ \\ ${ }^{1}$ School of Mathematics, Hefei University of Technology, Hefei 230009, China \\ ${ }^{2}$ School of Computer and Information, Hefei University of Technology, Hefei 23009, China \\ Correspondence should be addressed to Guangyue Tong; 1285036868@qq.com
}

Received 26 October 2020; Revised 2 December 2020; Accepted 18 December 2020; Published 6 January 2021

Academic Editor: Ghulam Mustafa

Copyright ( 2021 Jieqing Tan and Guangyue Tong. This is an open access article distributed under the Creative Commons Attribution License, which permits unrestricted use, distribution, and reproduction in any medium, provided the original work is properly cited.

This paper uses the continued fraction technique to construct a nonstationary 4-point ternary interpolatory subdivision scheme, which provides the user with a tension parameter that effectively handles cusps compared with a stationary 4-point ternary interpolatory subdivision scheme. Then, the continuous nonstationary 4-point ternary scheme is analyzed, and the limit curve is at least $C^{2}$-continuous. Furthermore, the monotonicity preservation and convexity preservation are proved.

\section{Introduction}

Subdivision schemes are wildly used in many areas, including CAGD, CG, and related areas. Most of the existing univariate subdivision schemes are binary, ternary, stationary, and linear. The classical binary 4-point scheme is one of the earliest and most popular interpolatory subdivision schemes $[1,2]$. Hassan et al. present the 4-point ternary subdivision scheme in [3], in which the limit curves by the 4-point ternary subdivision scheme are continuous. Beccari et al. present a nonstationary subdivision scheme in $[4,5]$, which generates continuous limit curves. In graphic design, shape-preserving of curve/surface is essential. Monotonicity preservation and convexity preservation are two significant properties in maintaining shape-preserving. Dyn et al. analyze the convexity preservation of the 4-point interpolatory scheme in [6]. Many subdivision schemes cannot satisfy monotonicity preservation and convexity preservation in the current subdivision zoo. Cai presents binary and ternary 4-point interpolatory subdivision schemes that are continuous in nonuniform control points and discusses the limit curve's convexity preservation [7, 8]. Kuijt and van Damme present a local nonlinear interpolatory subdivision scheme which is monotonicity preserving in [9], and Kuijt and van Damme also research a type of shape-preserving 4-point interpolatory subdivision scheme which interpolated nonuniform data in [10]. Tan et al. discuss the monotonicity preservation and convexity preservation of the binary subdivision scheme [11, 12]. Several subdivision schemes are designed to have their unique properties in [13-15]. Much research has been done on continued fraction theory and its application by Tan [16]. Ghaffar et al. discussed a new class of $2 \mathrm{q}$-point nonstationary subdivision schemes and their application [17]. Ashraf et al. analyzed the ternary four-point rational interpolating subdivision scheme's geometric properties [18, 19]. More subdivision schemes are studied in [17, 20-22].

Nonstationary subdivision schemes have been studied $[5,22]$, but the limit curves are not monotonicity preserving and convexity preserving. Our paper aims to construct a nonstationary 4-point ternary interpolatory subdivision scheme, which is shape-preserving using a continued fraction. Section 2 uses a continued fraction to construct a nonstationary interpolatory subdivision scheme and then analyze the continuity. In Section 3, the monotonicity preservation of the limit curves is discussed. In Section 4, the convexity preservation of limit curves is discussed and proven. In Section 5, when the initial control polygon is open, we use the new rule for the endpoints to achieve better continuity. In Section 6, we use experiments to show that our scheme effectively handles cusps and shape preservation. 


\section{A Nonstationary Subdivision}

\subsection{A Stationary Subdivision}

Definition 1 (see [3]). Given a set of the initial control points $P^{0}=\left\{p_{i}^{0} \in R^{d}\right\}_{i=1}^{n}$, let $P^{k}=\left\{p_{i}^{k} \in R^{d}\right\}_{i=1}^{n}$ be the set of control points at the order $k$ subdivision. Define $\left\{P_{i}^{k+1} \in R^{d}\right\}_{i=1}^{3^{k+1} n}$ recursively by the following ternary subdivision rules:

$$
\begin{cases}P_{3 i}^{k+1}=p_{i}^{k}, & 1<i<3^{k} n, \\ P_{3 i+1}^{k+1}=a_{0}^{k} p_{i-1}^{k}+a_{1}^{k} p_{i}^{k}+a_{2}^{k} p_{i+1}^{k}+a_{3}^{k} p_{i+2}^{k}, & 2<i<3^{k} n, \\ P_{3 i+2}^{k+1}=a_{3}^{k} p_{i-1}^{k}+a_{2}^{k} p_{i}^{k}+a_{1}^{k} p_{i+1}^{k}+a_{0}^{k} p_{i+2}^{k}, & 2<i<3^{k} n,\end{cases}
$$

where $a_{0}^{k} \equiv a_{0}=-1 / 18-1 / 6 u, a_{1}^{k} \equiv a_{1}=13 / 18+1 / 2 u, a_{2}^{k} \equiv$ $a_{2}=7 / 18-1 / 2 u$, and $a_{3}^{k} \equiv a_{3}=-1 / 18+1 / 6 u$. $u$ is a tension parameter. When $u \in(1 / 15,1 / 9)$, the limit curve is $C^{2}$-continuous.
2.2. A Nonstationary Subdivision. We use the continued fraction technique to construct a nonstationary 4-point ternary interpolatory subdivision scheme.

Definition 2. Given a set of the initial control points $P^{0}=\left\{p_{i}^{0} \in R^{d}\right\}_{i=1}^{n}$, let $P^{k}=\left\{p_{i}^{k} \in R^{d}\right\}_{i=1}^{n}$ be the set of control points at the order $k$ subdivision. Define $\left\{P_{i}^{k+1} \in R^{d}\right\}_{i=1}^{3^{k+1} n}$ recursively by the following ternary subdivision rules:

$$
\begin{cases}P_{3 i}^{k+1}=p_{i}^{k}, & 1<i<3^{k} n, \\ P_{3 i+1}^{k+1}=a_{0}^{k} p_{i-1}^{k}+a_{1}^{k} p_{i}^{k}+a_{2}^{k} p_{i+1}^{k}+a_{3}^{k} p_{i+2}^{k}, & 2<i<3^{k} n, \\ P_{3 i+2}^{k+1}=a_{3}^{k} p_{i-1}^{k}+a_{2}^{k} p_{i}^{k}+a_{1}^{k} p_{i+1}^{k}+a_{0}^{k} p_{i+2}^{k}, & 2<i<3^{k} n,\end{cases}
$$

where $a_{0}^{k}=-1 / 18-1 / 6 u^{k}, a_{1}^{k}=13 / 18+1 / 2 u^{k}, \quad a_{2}^{k}=7 / 18-$ $1 / 2 u^{k}$, and $a_{3}^{k}=-1 / 18+1 / 6 u^{k} . u^{k}$ is a tension parameter:

$$
\begin{aligned}
& u^{k}=b_{0}+\frac{c_{1} \mid}{\mid b_{1}}+\frac{c_{2} \mid}{\mid b_{2}}+\cdots+\frac{c_{k} \mid}{\mid b_{k}}, \\
& b_{0}=u+1, b_{j}=\frac{\left(u+1 / 4^{k+1}\right)\left(1+1 / 8^{k-1}\right)-\left(u+1 / 4^{k-1}\right)\left(1+1 / 8^{k+1}\right)}{\left(u+1 / 4^{k}\right)\left(1+1 / 8^{k-1}\right)-\left(u+1 / 4^{k-1}\right)\left(1+1 / 8^{k}\right)}, \\
& c_{j}=\frac{\left(u+1 / 4^{k-1}\right)\left(1+1 / 8^{k}\right)-\left(u+1 / 4^{k}\right)\left(1+1 / 8^{k+1}\right)}{\left(u+1 / 4^{k}\right)\left(1+1 / 8^{k-1}\right)-\left(u+1 / 4^{k-1}\right)\left(1+1 / 8^{k}\right)}, \quad u \in\left(\frac{1}{15}, \frac{1}{9}\right)(j=1,2, \ldots k) .
\end{aligned}
$$

Theorem 1 (Pringsheim Theorem [16]). Let $f_{n}=b_{0}+a_{1}|/| b_{1}+a_{2}|/| b_{2}+\cdots+a_{k}|/| b_{k}$, if $\left|b_{n}\right| \geq\left|a_{n}\right|+1$, so $f_{n}=b_{0}+a_{1}|/| b_{1}+a_{2}|/| b_{2}+\cdots+a_{k}|/| b_{k}$ is convergence.

Remark 1. According to Theorem 1, we know $u^{k}$ is convergence. Due to $u^{k}=b_{0}+c_{1}|/| b_{1}+c_{2}|/| b_{2}+\cdots+c_{k}|/| b_{k}$ $+\cdots=u+\left(1 / 4^{k+1}\right) / 1+\left(1 / 8^{k+1}\right)$, we know the $\lim _{k \longrightarrow+\infty} u^{k}=u, u \in(1 / 15,1 / 9)$.

2.3. Convergence Analysis. This section's purpose primarily proves the continuity of (2) when the initial tension parameter $u \in(1 / 15,1 / 9)$. To analyze our scheme's smoothness properties, we exploit Dyn and Levin's well-known results in [23], which relate the convergence of a nonstationary scheme to its asymptotically equivalent stationary counterpart. From Remark 1, we know $\lim _{k \longrightarrow+\infty} u^{k}=u, u \in(1 / 15,1 / 9)$, and the nonstationary subdivision scheme defined in (2) converges to the stationary subdivision scheme (1).

Proposition 1. The nonstationary subdivision scheme defined in (2) is asymptotically equivalent to the stationary scheme defined in (1) with $u \in(1 / 15,1 / 9)$. Moreover, it generates $C^{2}$-continuous limit curve.
Proof. If we want to prove that the proposed nonstationary subdivision scheme converges to a $C^{2}$-continuous limit curve, we compute its second divided difference mask and show that the associated limit curves are $C^{0}$-continuous. by

The mask of nonstationary subdivision scheme is given

$$
\begin{aligned}
m^{k}= & \frac{1}{18}\left[3 u^{k}-1,-3 u^{k}-1,0,-9 u^{k}+7,9 u^{k}+13,18,9 u^{k}\right. \\
& \left.+13,-9 u^{k}+7,0,-3 u^{k}-1,3 u^{k}-1\right]
\end{aligned}
$$

Its related first divided difference is

$$
\begin{aligned}
d_{(1)}^{k}= & \frac{1}{6}\left[3 u^{k}-1,-6 u^{k}, 3 u^{k}+1,-6 u^{k}+6,12 u^{k}+6,-6 u^{k}\right. \\
& \left.+6,3 u^{k}+1,-6 u^{k}, 3 u^{k}-1\right]
\end{aligned}
$$
be

Hence, the second divided difference mask turns out to 


$$
\begin{aligned}
d_{(2)}^{k}= & \frac{1}{2}\left[3 u^{k}-1,-9 u^{k}+1,9 u^{k}+1,-6 u^{k}\right. \\
& \left.+4,9 u^{k}+1,-9 u^{k}+1,3 u^{k}-1\right] .
\end{aligned}
$$

In this way, according to Remark 1, it follows that

$$
\begin{aligned}
\lim _{k \rightarrow \infty} d_{(2)}^{k}= & \frac{1}{2}[3 u-1,-9 u+1,9 u+1,-6 u+4,9 u+1, \\
& -9 u+1,3 u-1] .
\end{aligned}
$$

The mask $\lim _{k \rightarrow \infty} d_{(2)}^{k}$ tends to mask of the second divided differences of the stationary subdivision scheme defined in (1) with $u \in(1 / 15,1 / 9)$.

Since the stationary subdivision scheme is $C^{2}$-continuous when $u \in(1 / 15,1 / 9)$, then nonstationary subdivision scheme associated with $d_{(2)}^{\infty}$ will be $C^{0}$.

According to [16], if

$$
\sum_{k}^{+\infty}\left\|d_{(2)}^{k}-d_{(2)}^{\infty}\right\|_{\infty}<+\infty,
$$

then the two different schemes are asymptotically equivalent, so it concludes that the scheme associated with $d_{(2)}^{k}$ is $C^{0}$.

Since

$$
\begin{aligned}
\left\|d_{(2)}^{k}-d_{(2)}^{\infty}\right\|_{\infty} & =\frac{1}{2} \max \left\{\left|3 u^{k}-3 u\right|,\left|-9 u^{k}+9 u\right|,\right. \\
\left.\left|-6 u^{k}+6 u\right|\right\} & =\frac{9}{2}\left|u^{k}-u\right| .
\end{aligned}
$$

Next, we need to prove $9 / 2 \sum_{k=1}^{+\infty}\left|u^{k}-u\right|<+\infty$.

According to the structural characteristics of the $u^{k}$, we know $u^{k}>u^{k+1}$ and $\lim u^{k}=u, u \in(1 / 15,1 / 9)$. As $u^{k+1}<u^{k}, u^{k+1}-u>0, u^{k-+}-\dot{u}>0$, thus $u^{k+1}-u / u^{k}-u<1$, according to the comparison test, we know $\sum_{k=1}^{+\infty}\left|u^{k}-u\right|<+\infty$, so $9 / 2 \sum_{k=1}^{+\infty}\left|u^{k}-u\right|<+\infty$.

This completes the proof.

Hence, the nonstationary subdivision scheme defined in (2) is asymptotically equivalent to the stationary scheme defined in (1) with $u \in(1 / 15,1 / 9)$. Limit curves generated by (1) are $C^{2}$-continuous. According to Proposition 1 , the limit curves generated by (2) are $C^{2}$-continuous.

\section{Monotonicity Preservation}

The limit curves generated by (2) are $C^{2}$-continuous, we next discuss the limit curves generated by (2) are monotonicity preserving.

Proposition 2. Given a set of the initial control points $P^{0}=$ $\left\{p_{i}^{0} \in R^{d}\right\}_{i=1}^{n}$ that satisfies $\ldots p_{-1}^{0}<p_{0}^{0}<p_{1}^{0}<\ldots<p_{n-1}^{0}<p_{n}^{0}<\ldots$, a nonstationary subdivision scheme for designing curves generates a new control point $P^{k}=\left\{p_{i}^{k} \in R^{d}\right\}$ recursively at the level $k$ by applying (2). Denoting $D_{i}^{k}=p_{i+1}^{k}-p_{i}^{k}, q_{i}^{k}=$ $D_{i+1}^{k} / D_{i}^{k}, Q^{k}=\max \left\{q_{i}^{k}, 1 / q_{i}^{k}\right\}, \forall k \geq 0, k \in Z, i \in Z$. Furthermore, if $1 \leq \lambda \leq 4,{ }^{i} \lambda \in R, 1 / \lambda \leq Q^{0} \leq \lambda$, then

$$
D_{i}^{k}>0, \frac{1}{\lambda} \leq Q^{k} \leq \lambda, \quad \forall k \geq 0, k \in Z, i \in Z
$$

Proof. We use mathematic induction to verify Proposition 2. When $k=0, D_{i}^{0}=p_{i+1}^{0}-p_{i}^{0}>0,1 / \lambda \leq Q^{0} \leq \lambda$, then (10) is true.

Suppose that (10) is true for $k$, next we will verify it also holds true for $k+1$.

$$
\begin{aligned}
D_{3 i}^{k+1}= & p_{3 i+1}^{k+1}-p_{3 i}^{k+1}=\left(-\frac{1}{18}+\frac{1}{6} u^{k}\right)\left(p_{i+2}^{k}-p_{i+1}^{k}\right) \\
& +\left(\frac{6}{18}-\frac{2}{6} u^{k}\right)\left(p_{i+1}^{k}-p_{i}^{k}\right)+\left(\frac{1}{18}+\frac{1}{6} u^{k}\right)\left(p_{i}^{k}-p_{i-1}^{k}\right) \\
= & \left(-\frac{1}{18}+\frac{1}{6} u^{k}\right) D_{i+1}^{k}+\left(\frac{6}{18}-\frac{2}{6} u^{k}\right) D_{i}^{k}+\left(\frac{1}{18}+\frac{1}{6} u^{k}\right) D_{i-1}^{k} \\
= & D_{i}^{k}\left[\left(-\frac{1}{18}+\frac{1}{6} u^{k}\right) \frac{D_{i+1}^{k}}{D_{i}^{k}}+\left(\frac{6}{18}-\frac{2}{6} u^{k}\right)+\left(\frac{1}{18}+\frac{1}{6} u^{k}\right) \frac{D_{i-1}^{k}}{D_{i}^{k}}\right] \\
> & D_{i}^{k}\left[\left(-\frac{1}{18}+\frac{1}{6} u^{k}\right) \lambda+\left(\frac{6}{18}-\frac{2}{6} u^{k}\right)+\left(\frac{1}{18}+\frac{1}{6} u^{k}\right) \frac{1}{\lambda}\right] .
\end{aligned}
$$

As $1 \leq \lambda \leq 4, u^{k} \in(1 / 15,1 / 9)$, so

$$
\begin{aligned}
D_{3 i}^{k+1} & >D_{i}^{k}\left[\left(-\frac{1}{18}+\frac{1}{6} u^{k}\right) \times 4+\left(\frac{6}{18}-\frac{2}{6} u^{k}\right)+\left(\frac{1}{18}+\frac{1}{6} u^{k}\right)\right] \\
& =D_{i}^{k}\left(\frac{1}{6}+\frac{1}{2} u^{k}\right)>0 \\
D_{3 i+1}^{k+1} & =p_{3 i+2}^{k+1}-p_{3 i+1}^{k+1}=-\frac{2}{6} u^{k} D_{i+1}^{k}+\left(\frac{6}{18}+\frac{4}{6} u^{k}\right) D_{i}^{k}-\frac{2}{6} u^{k} D_{i-1}^{k} \\
& =D_{i}^{k}\left[-\frac{2}{6} u^{k} \frac{D_{i+1}^{k}}{D_{i}^{k}}+\left(\frac{6}{18}+\frac{4}{6} u^{k}\right)-\frac{2}{6} u^{k} \frac{D_{i-1}^{k}}{D_{i}^{k}}\right] \\
& >D_{i}^{k}\left[-\frac{2}{6} u^{k} \lambda+\left(\frac{6}{18}+\frac{4}{6} u^{k}\right)-\frac{2}{6} u^{k} \lambda\right]=D_{i}^{k}\left(\frac{4}{6} u^{k}(1-\lambda)+\frac{1}{3}\right)
\end{aligned}
$$

As $1 \leq \lambda \leq 4, u^{k} \in(1 / 15,1 / 9)$, so

$$
\begin{aligned}
D_{3 i+1}^{k+1}> & D_{i}^{k}\left(\frac{1}{3}-2 u^{k}\right)>0 \\
D_{3 i+2}^{k+1}= & p_{3 i+3}^{k+1}-p_{3 i+2}^{k+1}=\left(\frac{1}{18}+\frac{1}{6} u^{k}\right) D_{i+1}^{k}+\left(\frac{6}{18}-\frac{2}{6} u^{k}\right) D_{i}^{k} \\
& +\left(-\frac{1}{18}+\frac{1}{6} u^{k}\right) D_{i-1}^{k} \\
= & D_{i}^{k}\left[\left(\frac{1}{18}+\frac{1}{6} u^{k}\right) \frac{D_{i+1}^{k}}{D_{i}^{k}}+\left(\frac{6}{18}-\frac{2}{6} u^{k}\right)+\left(-\frac{1}{18}+\frac{1}{6} u^{k}\right) \frac{D_{i-1}^{k}}{D_{i}^{k}}\right] \\
> & D_{i}^{k}\left[\left(\frac{1}{18}+\frac{1}{6} u^{k}\right) \frac{1}{\lambda}+\left(\frac{6}{18}-\frac{2}{6} u^{k}\right)+\left(\frac{1}{18}+\frac{1}{6} u^{k}\right) \lambda\right]
\end{aligned}
$$

As $1 \leq \lambda \leq 4, u^{k} \in(1 / 15,1 / 9)$, so 


$$
\begin{array}{rlrl}
D_{3 i}^{k+1} & >D_{i}^{k}\left[\left(\frac{1}{18}+\frac{1}{6} u^{k}\right)+\left(\frac{6}{18}-\frac{2}{6} u^{k}\right)+\left(-\frac{1}{18}+\frac{1}{6} u^{k}\right) \times 4\right] & & \begin{array}{l}
\text { Now, we prove } 1 / \lambda \leq Q^{k} \leq \lambda . \\
\text { Since }
\end{array} \\
& =D_{i}^{k}\left(\frac{1}{6}+\frac{1}{6} u^{k}\right)>0 . &
\end{array}
$$

$$
\begin{aligned}
q_{3 i}^{k+1} & =\frac{D_{3 i+1}^{k+1}}{D_{3 i}^{k+1}}=\frac{-2 / 6 u^{k} D_{i+1}^{k}+\left(6 / 18+4 / 6 u^{k}\right) D_{i}^{k}-2 / 6 u^{k} D_{i-1}^{k}}{\left(-1 / 18+1 / 6 u^{k}\right) D_{i+1}^{k}+\left(6 / 18-2 / 6 u^{k}\right) D_{i}^{k}+\left(1 / 18+1 / 6 u^{k}\right) D_{i-1}^{k}} \\
& =\frac{-2 / 6 u^{k} q_{i+1}^{k}+\left(6 / 18+4 / 6 u^{k}\right)-2 / 6 u^{k} 1 / q_{i-1}^{k}}{\left(-1 / 18+1 / 6 u^{k}\right) q_{i+1}^{k}+\left(6 / 18-2 / 6 u^{k}\right)+\left(1 / 18+1 / 6 u^{k}\right) 1 / q_{i-1}^{k}}, \\
q_{3 i}^{k+1}-\lambda & =\frac{\left(-2 / 6 u^{k}+\lambda / 18-1 / 6 \lambda u^{k}\right) q_{i}^{k}+\left(6 / 18+4 / 6 u^{k}-6 \lambda / 18+2 / 6 \lambda u^{k}\right)-\left(2 / 6 u^{k}+\lambda / 18+1 / 6 \lambda u^{k}\right) 1 / q_{i-1}^{k}}{\left(-1 / 18+1 / 6 u^{k}\right) q_{i}^{k}+\left(6 / 18-2 / 6 u^{k}\right)+\left(1 / 18+1 / 6 u^{k}\right) 1 / q_{i-1}^{k}} .
\end{aligned}
$$

By (12), the denominator of the above expression is greater than zero. The numerator satisfies

$$
\begin{aligned}
\text { numerator } & <\left[\left(-2 / 6 u^{k}+\lambda / 18-1 / 6 \lambda u^{k}\right) \lambda+\left(6 / 18+4 / 6 u^{k}-6 \lambda / 18+2 / 6 \lambda u^{k}\right)-\left(2 / 6 u^{k}+\lambda / 18+1 / 6 \lambda u^{k}\right) 1 / \lambda\right] \\
& =\left(\lambda^{2} / 18-1 / 3 \lambda+5 / 18\right)+\left(-1 / 6 \lambda^{2}-1 / 3 \lambda+1 / 2\right) u^{k} .
\end{aligned}
$$

When $1 \leq \lambda \leq 4,-1 / 6 \lambda^{2}-1 / 3 \lambda+1 / 2 \leq 0, \lim _{k \longrightarrow+\infty} u^{k}=$ $u, u \in(1 / 15,1 / 9)$.
Then it can get numerator $<1 / 18\left(\lambda^{2}-6 \lambda\right.$ $+5)+\left(-1 / 6 \lambda^{2}-1 / 3 \lambda+1 / 2\right) \times 1 / 15 \leq 0$. So $q_{3 i}^{k+1}-\lambda \leq 0, q_{3 i}^{k+1}$ $\leq \lambda$.

In the same way, we prove the $1 / q_{3 i}^{k+1} \leq \lambda$.

$$
\frac{1}{q_{3 i}^{k+1}}-\lambda=\frac{\left(-1 / 18+1 / 6 u^{k}+2 / 6 u^{k}\right) q_{i}^{k}+\left(6 / 18-2 / 6 u^{k}-6 \lambda / 18-4 / 6 \lambda u^{k}\right)+\left(1 / 18+1 / 6 u^{k}+2 / 6 \lambda u^{k}\right) 1 / q_{i-1}^{k}}{-2 / 6 u^{k} q_{i+1}^{k}+\left(6 / 18+4 / 6 u^{k}\right)-2 / 6 u^{k} 1 / q_{i-1}^{k}}
$$

By (13), the denominator of the above expression is greater than zero. The numerator satisfies

$$
\begin{aligned}
\text { numerator } & <\left(-\frac{1}{18}+\frac{1}{6} u^{k}+\frac{2}{6} u^{k}\right) \lambda+\left(\frac{6}{18}-\frac{2}{6} u^{k}-\frac{6 \lambda}{18}-\frac{4}{6} \lambda u^{k}\right)+\left(\frac{1}{18}+\frac{1}{6} u^{k}+\frac{2}{6} \lambda u^{k}\right) \lambda \\
& =\frac{1}{3}\left[\left(2 \lambda^{2}-\lambda-1\right) u^{k}+(1-\lambda)\right] .
\end{aligned}
$$

When $1 \leq \lambda \leq 4, \quad 2 \lambda^{2}-\lambda-1 \geq 0, \lim _{k \longrightarrow+\infty} u^{k}=u, u \in$ $(1 / 15,1 / 9)$.

Then numerator $<1 / 3\left[\left(2 \lambda^{2}-\lambda-1\right) \times 1 / 9+(1-\lambda)\right]$ $=2 / 17\left(\lambda^{2}-5 \lambda+4\right) \leq 0$. So $1 / q_{3 i}^{k+1} \leq \lambda$.
Therefore, $1 / \lambda \leq q_{3 i}^{k+1} \leq \lambda, 1 / \lambda \leq 1 / q_{3 i}^{k+1} \leq \lambda$.

In the same way, we can prove $1 / \lambda \leq q_{3 i+1}^{k+1}$ $\leq \lambda, 1 / \lambda \leq 1 / q_{3 i+2}^{k+1} \leq \lambda$. So $\lambda \leq Q^{k+1}=\max \left\{q_{i}^{k+1}, 1 / q_{i}^{k+1}\right\} \leq \lambda$.

This completes the proof. 
Theorem 2 (see [10]). Given a set of initial control points $\left\{P_{i}^{0}\right\}_{i \in Z}$ which are strictly monotonically increasing (strictly monotone decreasing), such that $D_{i}^{0} \geq 0$.

Let

$$
\begin{aligned}
D_{i}^{k} & =p_{i+1}^{k}-p_{i}^{k}, q_{i}^{k}=\frac{D_{i+1}^{k}}{D_{i}^{k}}, Q^{k} \\
& =\max _{i}\left\{q_{i}^{k}, \frac{1}{q_{i}^{k}}\right\}, \quad \forall k \geq 0, k \in Z, i \in Z .
\end{aligned}
$$

Furthermore, the parameter $\lambda$ satisfies $1 / \lambda \leq Q^{0} \leq \lambda, 1 \leq \lambda \leq 4$, and the limit curves generated by (2) are strictly monotone increasing.

\section{Convexity Preservation}

Now we discuss the convexity preservation of the nonstationary subdivision scheme. We consider convexity preservation and convex control polygon so the limiting curve generated by our scheme preserves convexity initial data.

Definition 3 (see [23]). Given a set of control points $P^{0}=\left\{p_{i}^{0} \in R^{d}\right\}_{i=1}^{n}$, let $P^{k}=\left\{p_{i}^{k} \in R^{d}\right\}_{i=1}^{3^{n}}, p_{i}^{k}$ is strictly convex at a point $x_{i}^{k}$, if $f\left[x_{i-1}^{k}, x_{i}^{k}, x_{i+1}^{k}\right]>0$.

In this section, we check the convexity preservation of the nonstationary subdivision scheme (2) with uniform initial control points.

Given a set of initial control points $\left\{P_{i}^{0}\right\}_{i \in Z}, P_{i}^{0}=\left(x_{i}^{0}, p_{i}^{0}\right)$ which are strictly convex, where $\left\{x_{i}^{0}\right\}_{i \in Z}$ are equidistant points. For convenience, we make $\Delta x_{i}^{0}=x_{i+1}^{0}-x_{i}^{0}=1$. By the subdivision scheme (2), we have $\Delta x_{i}^{k+1}=x_{i+1}^{k+1}-x_{i}^{k+1}=1 / 3 \Delta x_{i}^{k}=1 / 3^{k+1}$. Denote $d_{i}^{k}=$ $f\left[x_{i-1}^{k}, x_{i}^{k}, x_{i+1}^{k}\right]=3^{2 k-1}\left(p_{i-1}^{k}-2 p_{i}^{k}+p_{i+1}^{k}\right)$ as the secondorder divided differences. In the following, we will prove $d_{i}^{k}>0, \forall k \geq 0, k \in Z, i \in Z$.

Proposition 3. Given that the initial control points $\left\{P_{i}^{0}\right\}_{i \in Z}, P_{i}^{0}=\left(x_{i}^{0}, p_{i}^{0}\right)$ are strictly convex, such that $d_{i}^{0}>0$, $\forall i \in Z, \quad$ denote $\quad r_{i}^{k}=d_{i+1}^{k} / d_{i}^{k}, R^{k}=\max \left\{r_{i}^{k}, 1 / r_{i}^{k}\right\}$, $\forall k \geq 0, k \in Z$. Furthermore, the parameter ${ }^{i} m$ satisfies
$1 \leq m \leq 2, m \in R$, then for $1 / m \leq R^{0} \leq m, P^{k}=\left\{p_{i}^{k} \in R^{d}\right\}$ recursively at the level $k$ by applying (2), then

$$
d_{i}^{k}>0, \frac{1}{m} \leq R^{k} \leq m, \quad \forall k \geq 0, k \in Z, i \in Z .
$$

Namely, the limit functions generated by the nonstationary subdivision scheme (2) are strictly convex.

Proof. We use mathematic induction to verify Proposition 3.

When $k=0, d_{i}^{0}>0,1 / m \leq R^{0} \leq m$, it is clear that (10) is true.

Suppose that (10) holds for $k$, next, we will verify it also holds for $k+1$.

$$
\begin{aligned}
d_{3 i}^{k+1} & =3^{2 k+1}\left(p_{3 i}^{k+1}-2 p_{3 i+1}^{k+1}+p_{3 i+2}^{k+1}\right) \\
& =3^{2 k+1}\left[\left(\frac{1}{18}+\frac{1}{2} u^{k}\right) d_{i-1}^{k}+\left(\frac{1}{18}-\frac{1}{2} u^{k}\right) d_{i}^{k}\right] .
\end{aligned}
$$

As $d_{i-1}^{k}>0, d_{i}^{k}>0, u^{k} \in(1 / 15,1 / 9)$, thus $d_{3 i}^{k+1}>0$.

$$
d_{3 i+1}^{k+1}=3^{2 k+1}\left[\left(\frac{1}{18}-\frac{1}{2} u^{k}\right) d_{i-1}^{k}+\left(\frac{1}{18}+\frac{1}{2} u^{k}\right) d_{i}^{k}\right] \text {. }
$$

As $d_{i-1}^{k}>0, d_{i}^{k}>0, u^{k} \in(1 / 15,1 / 9)$, thus $d_{3 i+1}^{k+1}>0$.

$$
\begin{aligned}
d_{3 i+2}^{k+1} & =3^{2 k+1}\left[\left(-\frac{1}{18}+\frac{1}{6} u^{k}\right) d_{i-1}^{k}+\left(\frac{4}{18}-\frac{2}{6} u^{k}\right) d_{i}^{k}+\left(-\frac{1}{18}+\frac{1}{6} u^{k}\right) d_{i+1}^{k}\right] \\
& =3^{2 k+1} d_{i}^{k}\left[\left(-\frac{1}{18}+\frac{1}{6} u^{k}\right) \frac{1}{r_{i}^{k}}+\left(\frac{4}{18}-\frac{2}{6} u^{k}\right)+\left(-\frac{1}{18}+\frac{1}{6} u^{k}\right) r_{i}^{k}\right] \\
& >3^{2 k+1} d_{i}^{k}\left[\left(-\frac{1}{18}+\frac{1}{6} u^{k}\right) m+\left(\frac{4}{18}-\frac{2}{6} u^{k}\right)+\left(-\frac{1}{18}+\frac{1}{6} u^{k}\right) m\right] \\
& =3^{2 k+1} d_{i}^{k}\left[\frac{1}{9}(2-m)+\frac{1}{3}(m-1) u^{k}\right] .
\end{aligned}
$$

As $1 \leq m \leq 2, u^{k} \in(1 / 15,1 / 9)$, thus $d_{3 i+2}^{k+1}>0$. Next we will prove $1 / m \leq R^{k+1} \leq m$.

Since

$$
\begin{aligned}
r_{3 i}^{k+1} & =\frac{d_{3 i+1}^{k+1}}{d_{3 i}^{k+1}}=\frac{\left(1 / 18-1 / 2 u^{k}\right) d_{i-1}^{k}+\left(1 / 18+1 / 2 u^{k}\right) d_{i}^{k}}{\left(1 / 18+1 / 2 u^{k}\right) d_{i-1}^{k}+\left(1 / 18-1 / 2 u^{k}\right) d_{i}^{k},} \\
r_{3 i}^{k+1}-m & =\frac{\left(1 / 18-1 / 2 u^{k}-m / 18+1 / 2 m u^{k}\right)+\left(\left(1 / 18+1 / 2 u^{k}-\left(m / 18+1 / 2 m u^{k}\right) r_{i}^{k}\right.\right.}{\left(\left(1 / 18+1 / 2 u^{k}\right)+\left(\left(1 / 18-1 / 2 u^{k}\right) r_{i}^{k}\right.\right.} .
\end{aligned}
$$

By (21), the denominator of (25) is greater than zero, and the numerator satisfies 


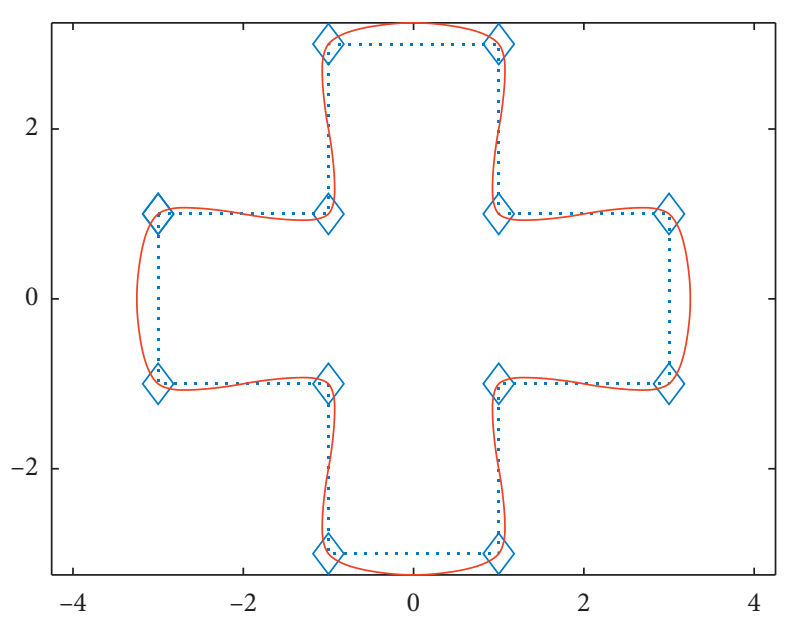

(a)

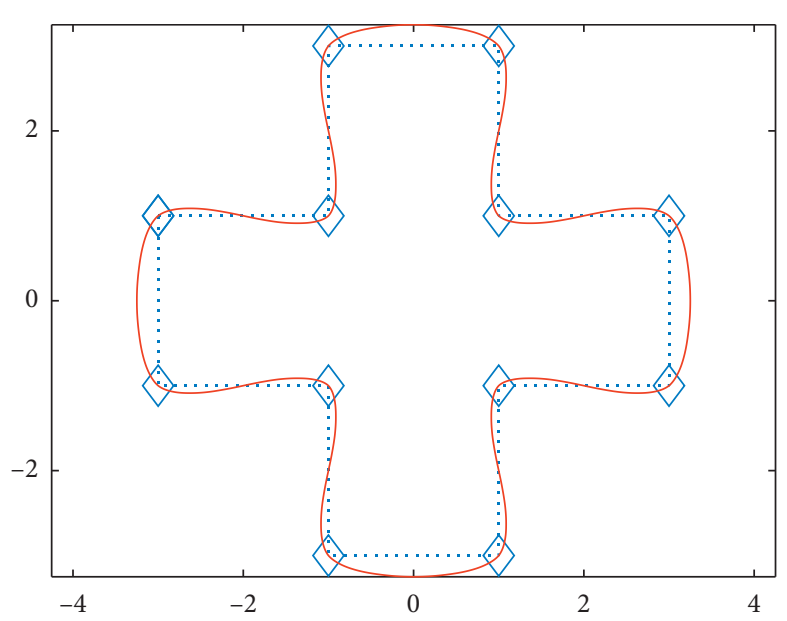

(c)

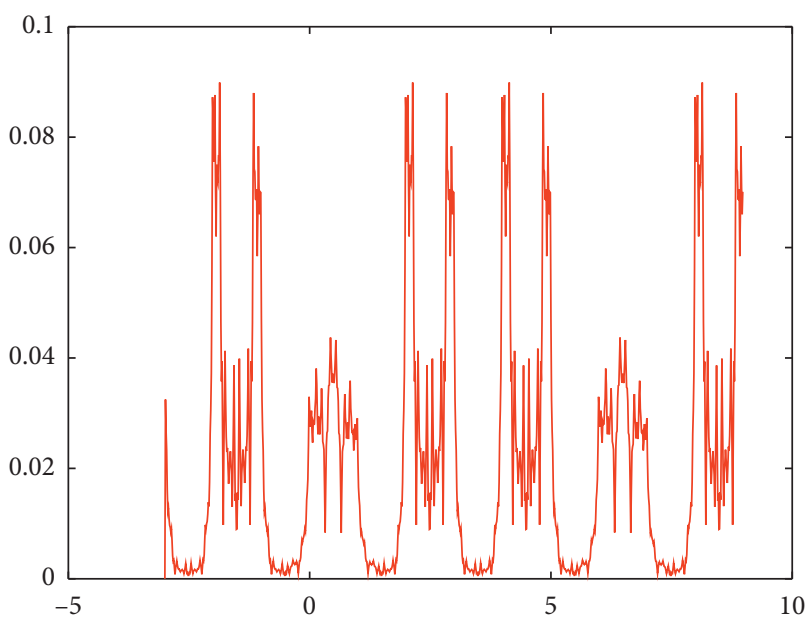

(b)

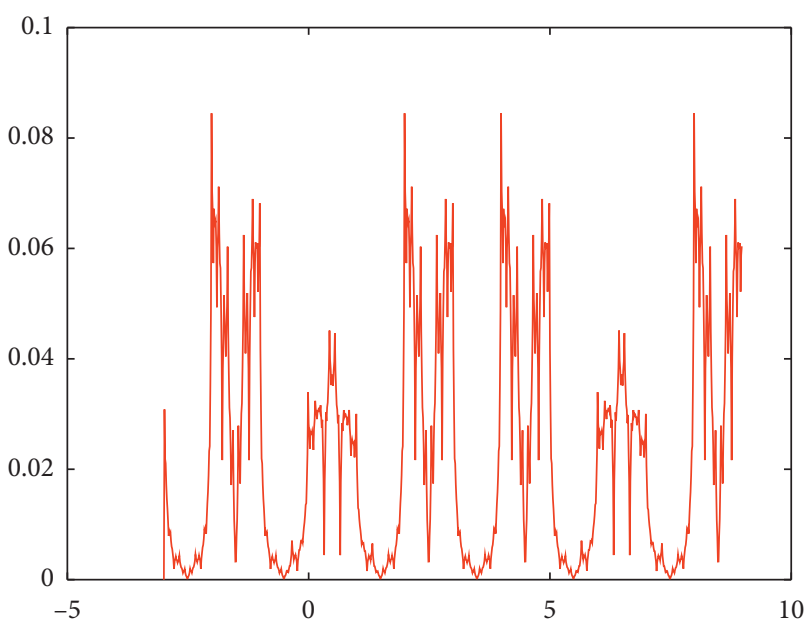

(d)

FIgURE 1: Limit curves and their curvature figures. (a) Stationary subdivision. (b) Curvature by (1). (c) Nonstationary subdivision. (d) Curvature by (2).

$$
\begin{aligned}
\text { numerator } & \leq\left(\frac{1}{18}-\frac{1}{2} u^{k}-\frac{1}{18} m-\frac{1}{2} u^{k} m\right)+\left(\frac{1}{18}+\frac{1}{2} u^{k}-\frac{m}{18}+\frac{1}{2} m u^{k}\right) m \\
& =-\frac{1}{18}\left(m^{2}-1\right)\left(1-9 u^{k}\right) .
\end{aligned}
$$

As $1 \leq m \leq 2, u^{k} \in(1 / 15,1 / 9)$, thus numerator $\leq 0$, so $r_{3 i}^{k+1} \leq m$.

In the same way, we can prove the $1 / r_{3 i}^{k+1} \leq m$.

$$
\frac{1}{r_{3 i}^{k+1}}-m=\frac{\left(1 / 18+1 / 2 u^{k}-m / 18+1 / 2 m u^{k}\right)+\left(1 / 18-1 / 2 u^{k}-m / 18-1 / 2 m u^{k}\right) r_{i}^{k}}{\left(1 / 18-1 / 2 u^{k}\right)+\left(1 / 18+1 / 2 u^{k}\right) r_{i}^{k}}
$$




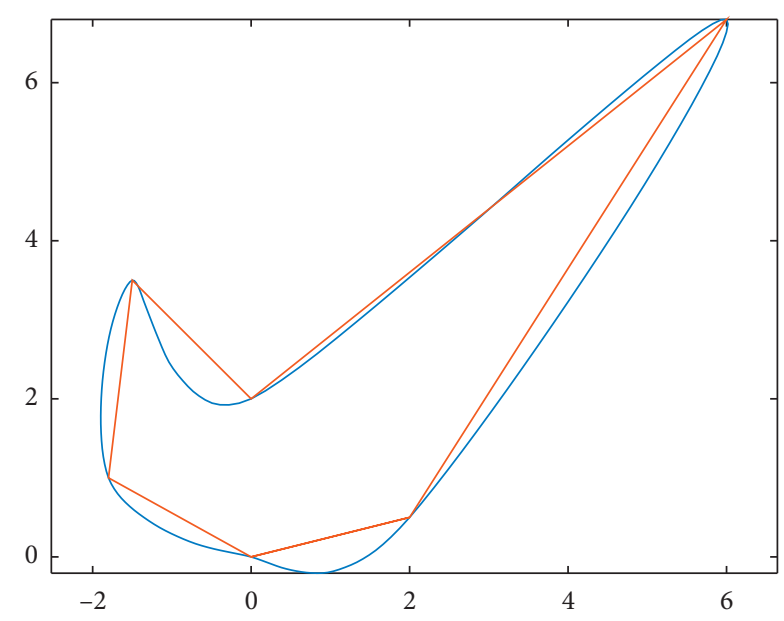

(a)

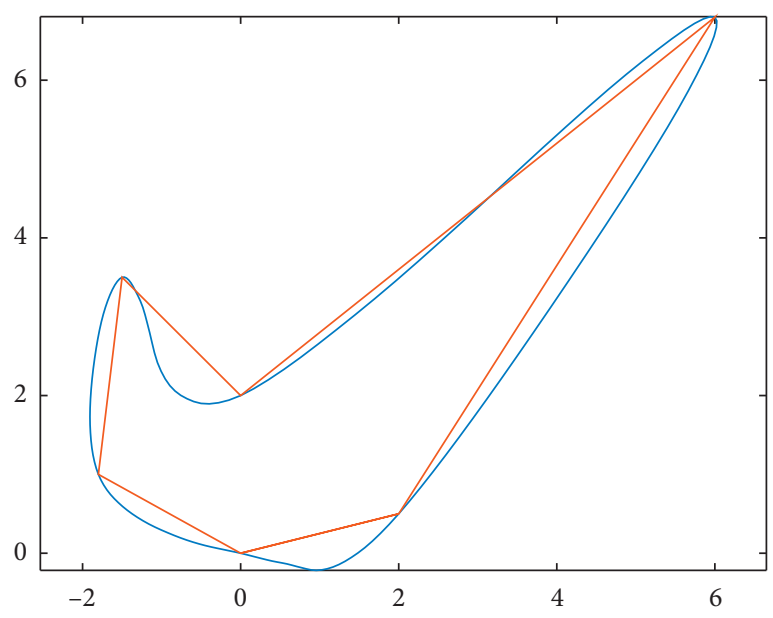

(c)

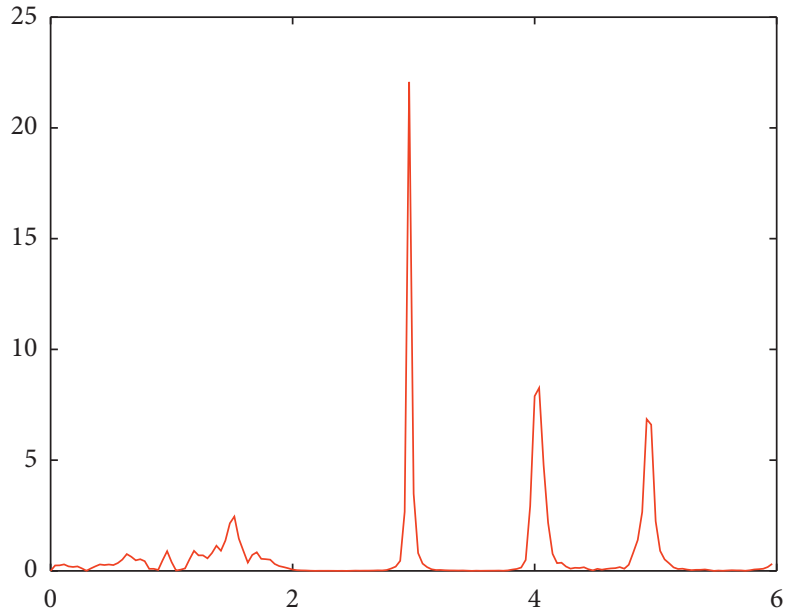

(b)

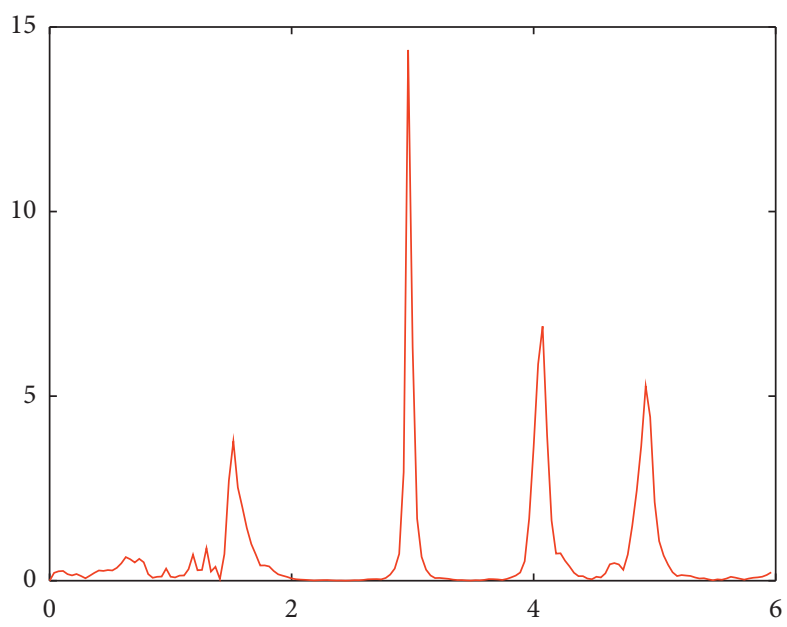

(d)

FIgURe 2: Limit curves and their curvature figures. (a) Stationary Nike by (1). (b) Curvature of stationary curvature (1). (c) nonstationary Nike by (2). (d) Curvature of stationary curvature (2).

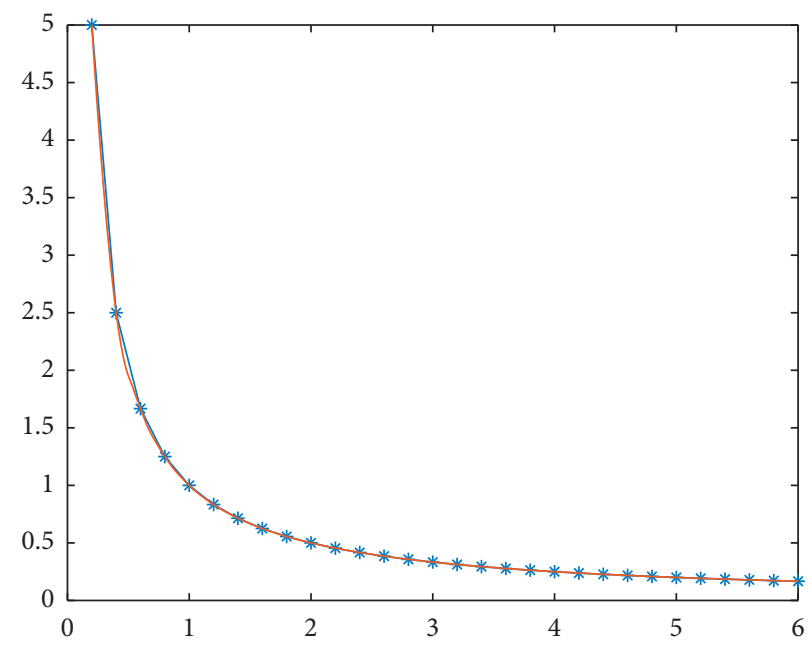

Figure 3: Limit curve by our scheme when $\lim _{k \rightarrow \infty} u_{k}=1 / 12$. The initial polygon derives from $f(x)=1 / x$. 


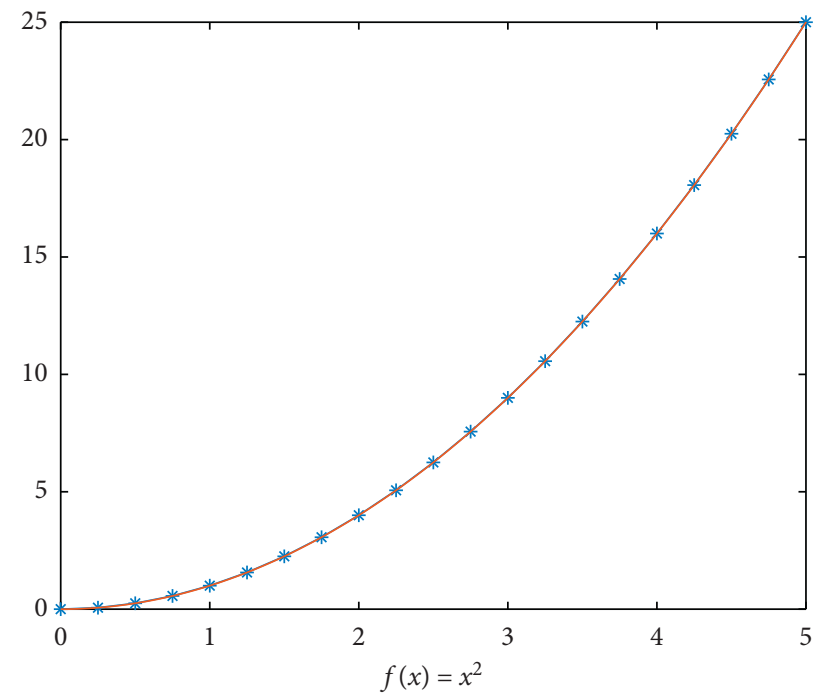

FIGURE 4: Limit curve by our scheme when $\lim _{k \rightarrow \infty} u_{k}=1 / 12$. The initial polygon derives from $f(x)=x^{2}$.

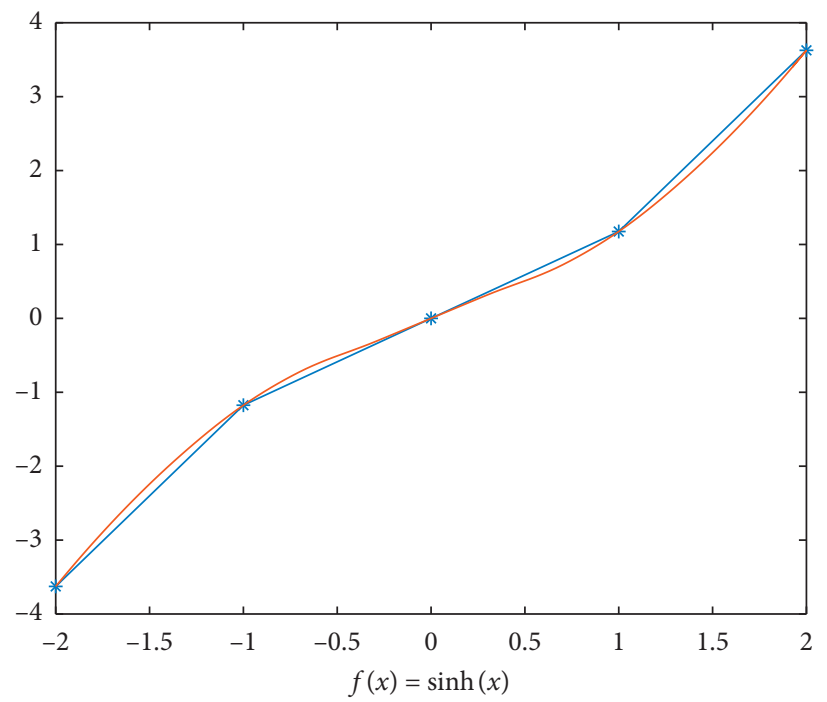

FIgURE 5: Limit curve by our scheme when $\lim _{k \rightarrow \infty} u_{k}=1 / 12$. The initial polygon derives from $f(x)=\sinh (x)$.

By (22), the denominator of (27) is greater than zero: numerator $\leq\left(\frac{1}{18}+\frac{1}{2} u^{k}-\frac{1}{18} m+\frac{1}{2} u^{k} m\right)+\left(\frac{1}{18}-\frac{1}{2} u^{k}-\frac{m}{18}-\frac{1}{2} m u^{k}\right) \frac{1}{m}$

$$
=-\frac{1}{18 m}\left(m^{2}-1\right)\left(1-9 u^{k}\right) \text {. }
$$

As $1 \leq m \leq 2, u^{k} \in(1 / 15,1 / 9)$, thus numerator $\leq 0$, so $1 / r_{3 i}^{k+1} \leq m$. Therefore, $1 / m \leq r_{3 i}^{k+1} \leq m$.

In the same way, we can prove $1 / m \leq r_{3 i+1}^{k+1}$ $\leq m, 1 / m \leq 1 / r_{3 i+1}^{k+1} \leq m, 1 / m \leq r_{3 i+2}^{k+1} \leq m, 1 / m \leq 1 / r_{3 i+2}^{k+1} \leq m$, so $1 / m \leq R^{k+1}=\max \left\{r_{i}^{k+1}, 1 / r_{i}^{k+1}\right\} \leq m$. This completes the proof.

From Proposition 3, the limit curves are generated by (2), which are convexity preserving.

\section{Improved Subdivision Interpolation Scheme at Endpoints}

If the initial control polygons are open, the new vertices near two endpoints cannot be calculated using (2), so we use the following subdivision schemes near the left endpoints and right endpoints to solve this problem.

The subdivision rules near the left endpoints are

$$
\left\{\begin{array}{l}
P_{1}^{k+1}=p_{i}^{k}, \\
P_{2}^{k+1}=\alpha_{0}^{k} p_{1}^{k}+\alpha_{1}^{k} p_{2}^{k}+\alpha_{2}^{k} p_{3}^{k}, \\
P_{3}^{k+1}=\beta_{0}^{k} p_{1}^{k}+\beta_{1}^{k} p_{2}^{k}+\beta_{2}^{k} p_{3}^{k} .
\end{array}\right.
$$

The subdivision rules near the right endpoints are 


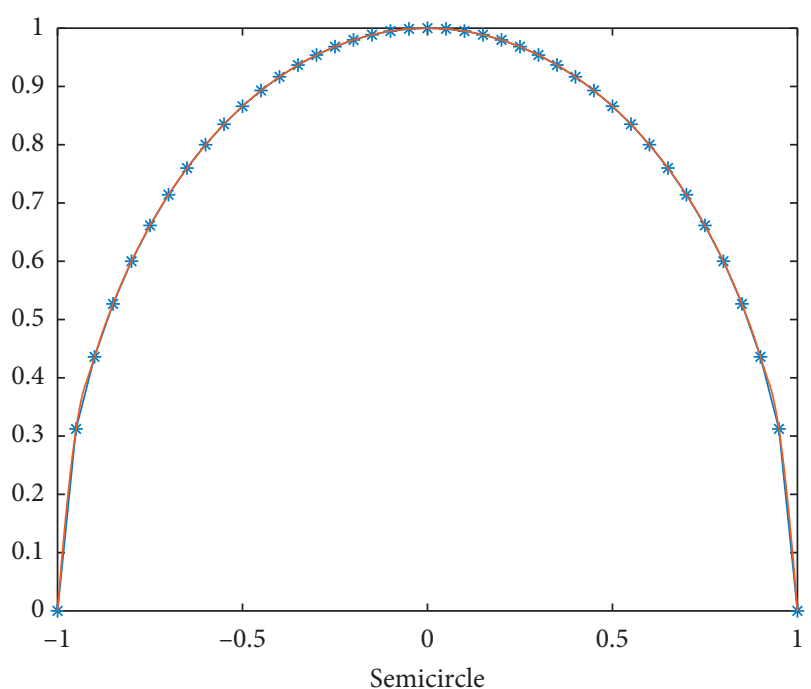

FiguRE 6: Limit curve by our scheme when $\lim _{k \rightarrow \infty} u_{k}=1 / 12$. The initial polygon derives from semicircle.

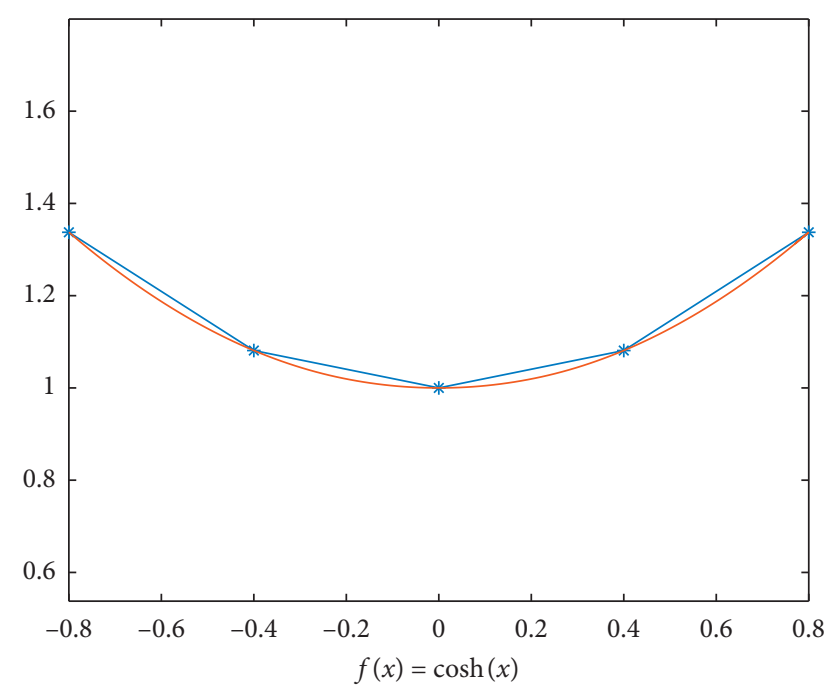

FIgURE 7: Limit curve by our scheme when $\lim _{k \longrightarrow \infty} \mathcal{u}_{k}=1 / 12$. The initial polygon derives from $f(x)=\cosh (x)$.

$$
\left\{\begin{array}{l}
P_{3^{k+1} n}^{k+1}=p_{3^{k} n}^{k}, \\
P_{3^{k+1} n-1}^{k+1}=\alpha_{0}^{k} p_{3^{k} n}^{k}+\alpha_{1}^{k} p_{3^{k} n-1}^{k}+\alpha_{2}^{k} p_{3^{k} n-2}^{k}, \\
P_{3^{k+1} n-2}^{k+1}=\beta_{0}^{k} p_{3^{k} n}^{k}+\beta_{1}^{k} p_{3^{k} n-1}^{k}+\beta_{2}^{k} p_{3^{k} n-2}^{k},
\end{array}\right.
$$

where

$$
\begin{aligned}
& \alpha_{0}^{k}=3 a_{0}^{k}+a_{1}^{k}, \alpha_{1}^{k}=a_{2}^{k}-3 a_{0}^{k}, \alpha_{2}^{k}=a_{0}^{k}+a_{3}^{k}, \\
& \beta_{0}^{k}=a_{1}^{k}+3 a_{3}^{k}, \beta_{1}^{k}=a_{1}^{k}-3 a_{3}^{k}, \beta_{2}^{k}=a_{0}^{k}+a_{3}^{k} .
\end{aligned}
$$

The improved interpolating subdivision scheme provides accurate endpoint interpolation. Wang and Qin in [24] have proved the limit curves by improved interpolating subdivision scheme are $C^{2}$-continuous when the masks are stationary scheme. However, according to Section 2, we can quickly get that (29) and (30) are $C^{2}$-continuous.

\section{Experiment and Conclusions}

This paper uses the continued fraction technique to construct a nonstationary 4-point ternary interpolatory subdivision scheme. The smoothness analysis is discussed, which indicates that the limit curve generated by the nonstationary subdivision scheme is continuous. Shapepreserving of the curve is essential. Monotonicity preservation and convexity preservation are two significant elements in shape-preserving. We have been proved that our schemes can ensure monotonicity preservation and convexity preservation when the conditions are imposed on the initial points in Sections 3 and 4. Nonstationary subdivision scheme by (2) and stationary subdivision scheme by (1) generate limit curves that are semblable by eye. So the approach of curvature plots is used to check out the efficiency.

The initial control polygons cannot satisfy monotonicity preservation and convexity preservation in Figures 1 and 2 . So the limit curves by our scheme are not shape-preserving. However, from Figure 1, the nonstationary subdivision scheme's curvature is better than the stationary subdivision scheme by (1); unfortunately, they are not obvious. Figure 2 shows that the shape of the curves and curvature of the nonstationary subdivision scheme by (2) are significantly better than those of the stationary subdivision scheme (1). By comparing the shape of the curve and curvature of Figure 1 with Figure 2, we can conclude that when the shapes of the control polygon are relatively flat, the shape and curvature of the nonstationary subdivision scheme by (2) are not significantly better than those of the stationary subdivision scheme by (1). Nevertheless, when the control polygon shapes are relatively steep or have cusps, the shape and curvature of the nonstationary subdivision scheme by (2) are significantly better than those of the stationary subdivision scheme by (1). Furthermore, Figures 3-5 show that if the initial control polygons are monotonicity preserving, our scheme's limit curves are also monotonicity preserving. From Figures 6 and 7, if the initial control polygons are convexity preserving, the limit curves are also convexity preserving by our scheme. So the experiments also show that our nonstationary subdivision scheme is shape-preserving.

\section{Data Availability}

All data used in this article are available from the corresponding author upon request.

\section{Conflicts of Interest}

The authors declare that they have no conflicts of interest.

\section{Acknowledgments}

This work was supported by the National Natural Science Foundation of China (NSFC) under the project nos. 61472466 and 61972131 . 


\section{References}

[1] S. Dubuc, "Interpolation through an iterative scheme," Journal of Mathematical Analysis and Applications, vol. 114, no. 1, pp. 185-204, 1986.

[2] N. Dyn, D. Levin, and J. A. Gregory, "A 4-point interpolatory subdivision scheme for curve design," Computer Aided Geometric Design, vol. 4, no. 4, pp. 257-268, 1987.

[3] M. F. Hassan, I. P. Ivrissimitzis, N. A. Dodgson, and M. A. Sabin, "An interpolating 4-point C2 ternary stationary subdivision scheme," Computer Aided Geometric Design, vol. 19, no. 1, pp. 1-18, 2002.

[4] C. Beccari, G. Casciola, and L. Romani, "An interpolating 4point ternary non-stationary subdivision scheme with tension control," Computer Aided Geometric Design, vol. 24, no. 4, pp. 210-219, 2007.

[5] C. Beccari, G. Casciola, and L. Romani, "A non-stationary uniform tension controlled interpolating 4-point scheme reproducing conics," Computer Aided Geometric Design, vol. 24, no. 1, pp. 1-9, 2007.

[6] N. Dyn, F. Kuijt, D. Levin, and R. van Damme, "Convexity preservation of the four-point interpolatory subdivision scheme," Computer Aided Geometric Design, vol. 16, pp. 789-792, 1987.

[7] Z. J. Cai, "Convergence, error estimation and some properties of four-point interpolation subdivision scheme," Computer Aided Geometric Design, vol. 12, pp. 459-468, 1995.

[8] Z. Cai, "Convexity preservation of the interpolating fourpoint ternary stationary subdivision scheme," Computer Aided Geometric Design, vol. 26, no. 5, pp. 560-565, 2009.

[9] F. Kuijt and R. van Damme, "Monotonicity preserving interpolatory subdivision schemes," Journal of Computational and Applied Mathematics, vol. 101, no. 1-2, pp. 203-229, 1999.

[10] F. Kuijt and R. van Damme, "Shape preserving interpolatory subdivision schemes for nonuniform data," Journal of Approximation Theory, vol. 114, no. 1, pp. 1-32, 2002.

[11] J. Tan, X. Zhuang, and L. Zhang, "A new four-point shapepreserving subdivision scheme," Computer Aided Geometric Design, vol. 31, no. 1, pp. 57-62, 2014.

[12] J. Tan, Y. Yao, H. Cao, and L. Zhang, "Convexity preservation of five-point binary subdivision scheme with a parameter," Applied Mathematics and Computation, vol. 245, pp. 279-288, 2014.

[13] M. A. Sabin and N. A. Dodgson, "A circle-preserving variant of the four-point subdivision scheme," in Mathematics Methods for Curves and Surfaces, Modern Methods in Mathematics, M. Daehlen, K. Morken, and L. L. Schumaker, Eds., pp. 275-286, Nashboro Press, Brentwood, TN, USA, 2004.

[14] C. Deng and G. Wang, "Incenter subdivision scheme for curve interpolation," Computer Aided Geometric Design, vol. 27, no. 1, pp. 48-59, 2010.

[15] C. Constanza and H. Kai, "Polynomial reproduction for univariate subdivision scheme of any arity," Journal of Approximation Theory, vol. 163, pp. 413-437, 2011.

[16] J. Q. Tan, Continued Fraction Theory and Its Application, pp. 35-36, Science Press, Beijing, China, 2007.

[17] A. Ghaffar, M. Bari, Z. Ullha, M. Iqbal, K. S Nisar, and D. Baleanu, "A new class of 2q-point non-stationary subdivision schemes and applications," Mathematics, vol. 7, p. 639, 2017.

[18] P. Ashraf, B. Nawaz, D. Baleanu et al., "Analysis of geometric properties of ternary four-point rational interpolating subdivision scheme," Mathematics, vol. 8, no. 3, p. 338, 2020.
[19] P. Ashraf, A. Ghaffar, D. Baleanu, I. Sehar, K. S. Nisar, and F. Khan, "Shape-preserving properties of a relaxed four-point interpolating subdivision scheme," Mathematics, vol. 8, no. 5, p. $806,2020$.

[20] L. Zou, L. Song, X. Wang, Y. Chen, C. Zhang, and C. Tang, "Bivariate thiele-like rational interpolation continued fractions with parameters based on virtual points," Mathematics, vol. 8, no. 1, p. 71, 2020.

[21] R. Hameed, G. Mustafa, A. Liaqat et al., "A new approach to increase the flexibility of curves and regular surfaces produced by 4-point ternary subdivision scheme," Mathematics Problems in Engineering, vol. 2020, Article ID 6096545, 17 pages, 2020.

[22] J. Tan, J. Sun, and G. Tong, "A non-stationary binary threepoint approximating subdivision scheme," Applied Mathematics and Computation, vol. 276, pp. 37-43, 2016.

[23] N. Dyn and D. Levin, "Analysis of asymptotically equivalent binary subdivision schemes," Journal of Mathematical Analysis and Applications, vol. 193, no. 2, pp. 594-621, 1995.

[24] H. Wang and K. Qin, "Improved ternary subdivision interpolation scheme," Tsinghua Science and Technology, vol. 10, no. 1, pp. 128-132, 2005. 